Michael J. Evans, Department of Mathematics, Washington and Lee

University, Lexington, Virginia 24450, e-mail: mjevans@wlu.edu

Paul D. Humke, Department of Mathematics, St. Olaf College, Northfield, Minnesota 45701, e-mail: humke@stolaf .edu

Richard J. O'Malley, Department of Mathematical Sciences, University of

Wisconsin, Milwaukee, Wisconsin 53201, e-mail: omalley@csd.uwm.edu

\title{
A PERPLEXING COLLECTION OF BAIRE ONE FUNCTIONS
}

\begin{abstract}
The problems of characterizing the class of universally polygonally approximable functions and the closure of that class are discussed.
\end{abstract}

\section{Introduction}

Our goals in writing this paper have been to: (1) Outline our current state of knowledge about universally polygonally approximable functions. (2) Point to several intriguing open problems and questions about this class.

Precise definitions of terms will be given in the next section. In the remainder of this section we provide a little background and try to get at the crux of the problem with as little technical baggage as possible. Clearly, a function $f:[0,1] \rightarrow \mathbb{R}$ belongs to Baire class one if and only if it is the pointwise limit of a sequence of polygonal (i.e., continuous, piecewise linear) functions. Agronsky, Ceder, and Pearson [1] asked the natural question, "Can we add the additional restriction that the vertices of the polygonal approximants lie on the graph of $f$ ?" Interestingly, they showed that this can, indeed, be done, thus adding to the collection of characterizations for this fundamental class. Now, what happens if we further ask that the approximants' vertices lie not only on the graph of $f$, but on the graph of $\left.f\right|_{C(f)}, f$ restricted to its set of continuity points? A moment's reflection, e.g. think of the characteristic function of a point, shows that this is not a vacuous restriction and that many Baire class one functions cannot be approximated in this way. The class of functions which can be so approximated is denoted UPA, and is the class of

Key Words: Baire one, polygonal approximation

Mathematical Reviews subject classification: 26A15

Received by the editors October 26, 1999 
functions referenced in the title of this paper. Before sketching why we are finding it perplexing, we note that if we add yet a third restriction, namely that the lengths of the linear segments of the polygonal functions go to zero, then the new collection is again not at all perplexing, being the known class of universally first return continuous functions [5], or equivalently, the quasicontinuous, Baire 1, Darboux functions [4]. Thus, on the surface, it would seem reasonable to assume that UPA has some natural characterization, as well. This may be true, but if so, it is elusive. There is both hidden structure and the lack of it to be found in UPA.

For example, it is straightforward to establish that if we can approximate $f$ via polygonal functions having vertices in $\left.f\right|_{C(f)}$, then actually we can construct approximants with vertices lying on the graph of $\left.f\right|_{D}$ for any preassigned dense set $D$. Thus, this is a fairly rich restriction and is the motivation for referring to this class as universally polygonally approximable or in short, UPA.

On the other hand, membership in UPA is fragile; UPA is not closed under truncation, addition, uniform convergence, or upon outer composition by

homeomorphisms. Some of these fragile natures were observed in [5]; others will be presented in the third section of this paper.

As UPA is not closed under uniform convergence, one might hope that its closure has a natural characterization and in the next section we offer a candidate for the closure of UPA. But although this candidate class is closed and contains UPA, our conjecture that UPA is dense in that class remains an open problem. Thus, while we have quite a bit of information about the nature of individual UPA functions, the two fundamental problems of characterizing the class UPA and its closure remain open.

The following section is devoted to giving several definitions which are then used in the third section and fourth sections, where we prove a few new results, weave these together with results published elsewhere, and detail the remaining open questions.

\section{Definitions and Notation}

Unless otherwise stated, functions considered here are real-valued and defined on the interval $[0,1]$.

Definition 1. Let $f:[0,1] \rightarrow \mathbb{R}$.

a) We say that a function $h:[0,1] \rightarrow \mathbb{R}$ is a polygonal function for $f$ if there is a partition $\tau=\left\{0=a_{0}<a_{1}<a_{2}<\cdots<a_{m}=1\right\}$ such that $h$ agrees with $f$ at each partition point and is linear on the intervening closed intervals. We call $a_{0}, a_{1}, \ldots, a_{m}$ the nodes of $h$ and 
$\left(a_{0}, h\left(a_{0}\right)\right),\left(a_{1}, h\left(a_{1}\right)\right), \ldots,\left(a_{m}, h\left(a_{m}\right)\right)$ the vertices of $h$. The maximum distance between adjacent nodes is called the mesh of $h$ and the maximum distance between adjacent vertices is called the graph-mesh of $h$. These are denoted mesh $(h)$ and graph-mesh $(h)$, respectively.

b) If $S \subset[0,1]$, we say that a sequence $\left\{h_{n}\right\}$ of polygonal functions for $f$ polygonally approximates $f$ on $S$ if $\lim _{n \rightarrow \infty} h_{n}(x)=f(x)$ for every $x \in S$ and $\lim _{n \rightarrow \infty}$ mesh $\left(h_{n}\right)=0$. In this case we say that $f$ is polygonally approximable on $S$, and if $S=[0,1]$ we say that $f$ is polygonally approximable. Further, if all the nodes of the polygonal functions, other than 0 and 1, belong to the set of points of continuity, $C(f)$, we say that $\left\{h_{n}\right\} C(f)$-polygonally approximates $f$.

c) If graph-mesh $\left(h_{n}\right)$ replaces mesh $\left(h_{n}\right)$ in b) then we obtain the notion of a strongly polygonally approximable function.

Definition 2. Let $f:[0,1] \rightarrow \mathbb{R}$. We shall say that

a) $f$ is universally polygonally approximable if for every dense subset $D$ in $[0,1]$ there is a sequence $\left\{h_{n}\right\}$ of polygonal functions for $f$, having nodes in $D \cup\{0,1\}$ which polygonally approximates $f$ on $[0,1]$. We let $U P A$ denote the set of all universally polygonally approximable functions.

b) $f$ is strongly universally polygonally approximable if for every dense subset $D$ in $[0,1]$ there is a sequence $\left\{h_{n}\right\}$ of polygonal functions for $f$, having nodes in $D \cup\{0,1\}$ which strongly polygonally approximates $f$ on $[0,1]$.

Definition 3. A function $f:[0,1] \rightarrow \mathbb{R}$ is quasi-continuous at $x$ if every neighborhood of $(x, f(x))$ contains a point of $f \mid C(f)$. We let $Q(f)$ denote the set of points of quasi-continuity of $f$ and $N Q(f)=[0,1] \backslash Q(f)$. If $Q(f)=[0,1]$, we say that $f$ is a quasi-continuous function and we let $Q C$ denote the class of all such functions.

Although far from being sufficient, the following property is clearly necessary for a function to belong to UPA.

Definition 4. We say that the function $f:[0,1] \rightarrow \mathbb{R}$ is interpolatively continuous at $x \in(0,1)$ if for each $\epsilon>0$ there exist points $y, z$ in $C(f)$ such that

$$
x-\epsilon<y<x<z<x+\epsilon \text { and }\left|L_{y z}(x)-f(x)\right|<\epsilon,
$$

where $L_{y z}(x)$ denotes the linear function passing through the points $(y, f(y))$ and $(z, f(z))$. More generally, we let $L_{(s, t)}^{(u, v)}$ denote the linear function passing 
through the two points $(s, t),(u, v)$. Thus, when a function $f$ is understood, $L_{y z}$ supplants the more cumbersome $L_{(y, f(y))}^{(z, f(z))}$. We let $I C(f)$ denote the set of points at which $f$ is interpolatively continuous. If $I C(f)=(0,1)$, we say that $f$ is an interpolatively continuous function and let $I C$ denote the class of all such functions.

If $f:[0,1] \rightarrow \mathbb{R}$ and $\tau=\left\{a=a_{0}<a_{1}<a_{2}<\cdots<a_{n}=b\right\}$ is a partition of $[a, b]$, we let $P_{f, \tau,[a, b]}$ denote that function which agrees with $f$ at each point of $\tau$ and is linear on the intervening intervals. We omit the subscript $f$ if the function $f$ is understood, and if $a=0$ and $b=1$, we let $P_{\tau}=P_{\tau,[0,1]}$. Armed with this notation, we define a notion somewhat stronger than interpolative continuity:

Definition 5. A function $f:[0,1] \rightarrow \mathbb{R}$ is said to have the delta-fine property if for each closed set $W$ and each $\epsilon>0$, there are two points $a<b$ in $C(f)$ such that $[a, b] \cap W \neq \emptyset$ and such that for every $\delta>0$ there is a $\delta$-fine partition $\tau=\left\{a=a_{0}<a_{1}<a_{2}<\cdots<a_{n}=b\right\}$ of $[a, b]$ consisting of points in $C(f)$ such that $\left|P_{\tau,[a, b]}(x)-f(x)\right|<\epsilon$ for every $x \in W \cap[a, b]$. We let $D F$ denote the class of all functions having the delta-fine property.

In [5] UPA functions were characterized in terms of their local behavior; namely, $f \in \mathrm{UPA}$ if and only if for each closed set $E$ there is a portion $(c, d) \cap E$ on which $f$ is $C(f)$-polygonally approximable. We also show in [5] that the class UPA is not closed in the sup metric. Our search for a characterization of the closure of UPA led to the class DF defined above. It is relatively straightforward to see that UPACDF and that DF is closed in the sup metric (see Propositions 6 and 7 of this paper). It would seem that $\mathrm{DF}$ is a good candidate for the closure of UPA, but whether or not $\mathrm{DF}=\overline{\mathrm{UPA}}$ remains open.

One final reminder of terminology before moving to the next section:

Definition 6. We shall use $\omega_{f}(x)$ to denote the oscillation of $f$ at $x$; that is,

$$
\omega_{f}(x)=\lim _{r \downarrow 0}[\sup \{f(t):|t-x|<r\}]-\lim _{r \downarrow 0}[\inf \{f(t):|t-x|<r\}] .
$$

The oscillation of $f$ over a set $S$ will be denoted $\omega_{f}(S)$ and is given by

$$
\omega_{f}(S)=\sup \{|f(u)-f(v)|: u, v \in S\} .
$$

\section{Some UPA Functions}

In this section we will first go to some length to construct a non-negative UPA function whose square is not UPA and mention some consequences. Then we show that certain familiar classes of functions are in UPA or its closure. 
A function such as

$$
f(x)= \begin{cases}-1 & \text { if } x<1 / 2 \\ 0 & \text { if } x=1 / 2 \\ 1 & \text { if } x>1 / 2\end{cases}
$$

is easily seen to belong to UPA, while $f^{2} \notin \mathrm{UPA}$; indeed, $f^{2}$ is not even interpolatively continuous. It is considerably more challenging to think of a non-negative such $f$. Our primary reason for wanting a non-negative example is because it will show that, upon viewing $h:[0, \infty) \rightarrow[0, \infty)$, given by $h(x)=$ $x^{2}$, as a homeomorphism, UPA is not closed under the application of outer homeomorphisms, a situation reminiscent of the class of derivatives, another perplexing collection of Baire one functions. Then, following the argument employed by Bruckner [2, p. 70, for example], we may conclude that UPA cannot be characterized by associated sets. Thus, the effort we are about to expend on developing such an example seems justified. Our construction will take advantage of a certain Cantor set $T$ described in [5]. To facilitate reading, we include a description of that Cantor set here.

The set $T$ is constructed inductively in stages by removing at stage $n$ a finite collection $\mathcal{U}_{n}$ of relatively large open intervals from $[0,1]$; indeed, at stage $n$ we shall be removing $\frac{n}{n+1}$ of the remaining measure.

At stage 1 we delete the collection $\mathcal{U}_{1}$ consisting of 4 equally spaced open intervals, each of length $l_{1}=1 / 8$. We let $U_{1}$ denote the union of those deleted intervals and set $T_{1}=[0,1] \backslash U_{1}$. Thus the components of $T_{1}$ form a collection $\mathcal{T}_{1}$ of 5 closed intervals, each of length $g_{1}=1 / 10$.

Next, assume that stage $n$ has been completed. That is, we assume that all of the following have been identified:

- the collection $\mathcal{U}_{n}$ of $4 \cdot 5^{n-1}$ open intervals, each of length

$$
l_{n}=\frac{1}{4} \cdot \frac{1}{5^{n-1}} \cdot \frac{n}{(n+1) !}
$$

- the collection $\mathcal{T}_{n}$ of $5^{n}$ closed intervals, each of length

$$
g_{n}=\frac{1}{5^{n}} \cdot \frac{1}{(n+1) !}
$$

- the open set $U_{n}$ as the union of the sets in $\mathcal{U}_{n}$,

- and the closed set $T_{n}$ as the union of the sets in $\mathcal{T}_{n}$. 
To perform stage $(n+1)$, from each component of $T_{n}$ we delete 4 equally spaced and centrally located open intervals, each of length

$$
l_{n+1}=\frac{1}{4} \cdot \frac{n+1}{n+2} \cdot g_{n}=\frac{1}{4} \cdot \frac{1}{5^{n}} \cdot \frac{n+1}{(n+2) !},
$$

leaving 5 closed intervals remaining in this component, each having length

$$
g_{n+1}=\frac{1}{5} \cdot\left(g_{n}-4 l_{n+1}\right)=\frac{1}{5^{n+1}} \cdot \frac{1}{(n+2) !} .
$$

We let $\mathcal{U}_{n+1}$ denote the collection of the open intervals of length $l_{n+1}$ removed at this stage and let $U_{n+1}$ denote the open set formed as the union of the elements of $\mathcal{U}_{n+1}$. We let $T_{n+1}=[0,1] \backslash \bigcup_{k=1}^{n+1} U_{k}$, and denote the collection of the $5^{n+1}$ components of $T_{n+1}$ as $\mathcal{T}_{n+1}$, noting that each element of $\mathcal{T}_{n+1}$ is a closed interval of length $g_{n+1}$. This completes stage $(n+1)$.

Finally, we set $T=\bigcap_{n=1}^{\infty} T_{n}$. Using the symmetry of this construction and the fact that $\lim _{n \rightarrow \infty} g_{n} / l_{n}=0$ it is easy to see that $T$ has symmetric porosity one at each of its points. A set having symmetric porosity one at each of its points is called strongly symmetrically porous. Although we will not be explicitly using any notion of porosity in the following construction, the strongly symmetric porosity of $T$ will indirectly allow use to achieve our goal. Not only is the above limit of significance, but so is the fact that $n g_{n}<l_{n}$.

Example 1. There is a UPA function $F:[0,1] \rightarrow[0, \infty)$ for which $F^{2}$ is not UPA.

Proof. Let $T$ be the Cantor set described above and first assign $F$ the value 1 at each point of $T$. We shall define the function $F$ on $[0,1] \backslash T$, by assigning its values on the contiguous intervals inductively. We assign $F$ the values $(0,2,0,2)$ in that order from left to right on the 4 open intervals in $\mathcal{U}_{1}$. Now assume that $F$ has been assigned values on each of the intervals in $\cup_{k=1}^{n} \mathcal{U}_{k}$. We now assign $F$ a value of 0 or $\sqrt{n+1}+1$ on each of the $4 \cdot 5^{n}$ intervals in $\mathcal{U}_{n+1}$ by treating them in sets of four, each set consisting of the four open intervals removed from a closed interval in $\mathcal{I}_{n}$. Let $\mathcal{I}$ denote such a collection of four intervals in $\mathcal{U}_{n+1}$. If $\mathcal{I}$ is the leftmost or the rightmost such collection, we assign $f$ the values $(0, \sqrt{n+1}+1,0, \sqrt{n+1}+1)$ in that order from left to right on the 4 intervals in $\mathcal{I}$. For any other $\mathcal{I}$ we look at the nearest intervals, $L$ and $R$, from the collection $\cup_{k=1}^{n} \mathcal{U}_{k}$, lying to the left and right, respectively, of $\mathcal{I}$. Note that $F$ has previously been assigned a value of 0 on $L$ and a positive value on $R$, or vice versa. If the former situation holds, we assign $F$ the values $(\sqrt{n+1}+1,0, \sqrt{n+1}+1,0)$ in that order from left to right on the four intervals composing $\mathcal{I}$. If the latter situation holds, we assign $F$ the 
values $(0, \sqrt{n+1}+1,0, \sqrt{n+1}+1)$ in that order from left to right on the four intervals composing $\mathcal{I}$. This completes the definition of $F$.

Let us next show that $F$ is universally polygonally approximable. We shall construct the $n$th polygon $h_{n}$ so that all of its nodes lie in $\cup_{k=1}^{n} U_{k}$. In fact, the nodes of the polygons will consist of 0,1 , and two points from each interval in $\cup_{k=1}^{n} U_{k}$, each lying within a distance of $n g_{n} / 2$ to the nearest endpoint of that interval. This information alone assures that $\left\{h_{n}\right\}$ will converge to $F$ pointwise on $[0,1] \backslash T$. The delicate part of the definition of the $h_{n}$ 's is to guarantee that $\left\{h_{n}(x)\right\}$ converges to 1 at each point $x \in T$, while satisfying the above conditions. We next turn our attention to accomplishing this by carefully defining $h_{n}$.

Let $n \in \mathbb{N}$. We shall first define how we wish $h_{n}$ to behave on each closed interval in $\mathcal{T}_{n}$. Let $[a, b]$ be such an interval. Let $L$ and $R$ be the two intervals in $\cup_{k=1}^{n} \mathcal{U}_{k}$ abutting $[a, b]$ on the left and right, respectively. now, $F$ has value zero on one of $L$ and $R$ and positive value less than or equal to $\sqrt{n}+1$ on the other. For specificity, we will consider the case where $F$ is zero on $L$. (The other case is handled via a symmetric argument.) Let $\ell$ be the function defined on $[a, b]$ whose graph is the line segment with endpoints $(a, 1-1 / \sqrt{n})$ and $(b, 1+1 / \sqrt{n})$. Note that the slope of $\ell$ is $2 /\left(\sqrt{n} g_{n}\right)$.

Linearly extend the graph of $\ell$ to the left, maintaining slope $2 /\left(\sqrt{n} g_{n}\right)$ until the extended line segment hits the $x$-axis at some point $(a-s, 0)$. We may compute $s$ as follows:

$$
s=\frac{\sqrt{n} g_{n}}{2}\left(1-\frac{1}{\sqrt{n}}\right)=\frac{\sqrt{n}-1}{2} g_{n}<\frac{n g_{n}}{2},
$$

indicating that $a-s$ lies in the right half of $L$; indeed it is within $n g_{n} / 2$ of the right endpoint, as desired. The point $a-s$ becomes a node for $h_{n}$.

Next, linearly extend the graph of $\ell$ to the right, maintaining slope $2 /\left(\sqrt{n} g_{n}\right)$ until the extended line segment, call it $\ell^{*}$, hits the line $y=\sqrt{n}+1$ at some point $(b+t, 0)$. Computing $t$, we have

$$
t=\frac{\left(\sqrt{n}-\frac{1}{\sqrt{n}}\right) \sqrt{n} g_{n}}{2}=\frac{n-1}{2} g_{n}<\frac{n g_{n}}{2},
$$

indicating that $b+t$ is in the left half of $R$, lying within $n g_{n} / 2$ of the left endpoint of $R$. Let $t^{*}$ be the smallest positive number such that $\ell^{*}\left(b+t^{*}\right)=$ $F\left(b+t^{*}\right)$. Thus, $t^{*} \leq t$ and so $b+t^{*}$ also is in the left half of $R$, lying within $n g_{n} / 2$ of the left endpoint, as desired. We let $b+t^{*}$ be a node of $h_{n}$.

In this manner, each $[a, b] \in \mathcal{T}_{n}$, except the leftmost and rightmost, gives rise to two nodes in its corresponding $L$ and $R$. 
The leftmost interval in $\mathcal{T}_{n}$ is $\left[0, g_{n}\right]$ and for this interval we take $\ell$ to be the function on $\left[0, g_{n}\right]$ whose graph is the line segment having endpoints $(0,1)$ and $(b, 1-2 / \sqrt{n}$. Extending this line segment to the right, we see that it hits the $x$-axis at $g_{n}+t$, where

$$
t=\frac{\sqrt{n}-2}{2} g_{n} \frac{n g_{n}}{2} .
$$

We make 0 and $g_{n}+t$ be the nodes resulting from this leftmost $[a, b]$.

For the rightmost $[a, b] \in \mathcal{T}_{n}$ we have $[a, b]=\left[1-g_{n}, 1\right]$ and we take $\ell$ to be the function on $[a, b]$ whose graph is the line segment having endpoints $(1,1)$ and $(a, 1+2 / \sqrt{n})$. Extending this line segment to the left, we see that it hits the line $y=\sqrt{n}+1$ at $a-s$, where

$$
s=\frac{\sqrt{n}-2}{2} g_{n} \frac{n g_{n}}{2} .
$$

We make 1 and $a-s$ be the nodes resulting from this rightmost $[a, b]$.

Now $h_{n}$ is completely determined by these $2 \cdot 5^{n}$ nodes and we have that for each $x \in T_{n},\left|h_{n}(x)-1\right|<2 / \sqrt{n}$, implying that for each $x \in T$, we have $\left|h_{n}(x)-F(x)\right|<2 / \sqrt{n}$. Recall that the pointwise convergence of $h_{n}$ to $F$ on the complement of $T$ is assured by the fact that we have made the 2 nodes of $h_{n}$ that occur in any fixed $I \in \mathcal{U}_{k}, k<n$, migrate towards the endpoints of $I$. Thus $F$ is UPA.

To see that $F^{2}$ is not universally polygonally approximable, suppose to the contrary that there is a sequence $\left\{h_{n}\right\}$ of polygonal functions which converges pointwise to $F^{2}$ and for which all of the vertices are on the graph of $F^{2}$ with first coordinates in $C\left(F^{2}\right)$. Since $\left\{h_{n}\right\}$ converges to $F^{2}$ pointwise on $T$, according to the Baire Category Theorem, there must be what a number of authors call a point of uniform convergence of $\left\{h_{n}\right\}$. That is, there is a point $p \in T$ such that for each $\epsilon>0$ there is a portion $T_{\epsilon}$ of $T$, containing $p$, and an $N_{\epsilon}$ such that for all $n>N_{\epsilon}$ and all $x \in T_{\epsilon},\left|h_{n}(x)-F^{2}(x)\right|<\epsilon$. Consider, specifically, the situation where $\epsilon=0.01$ and let $T_{0.01}=(a, b) \cap T$. Let $I$ be a contiguous interval to $T$ such that $I \subset(a, b), F^{2}(I)=\{0\}$, and $T_{0.01}$ has points to the right of $I$. Choose $k$ such that $I \in \mathcal{U}_{k}$. Now, there exists an $n>N_{0.01}$ and $J \in \mathcal{U}_{j}$ for some $j>k$ such that $J \subset(a, b), J$ lies to the right of $I$, and the polygonal function $h_{n}$ contains a line segment which has its left endpoint $(\alpha, 0) \in I \times\{0\}$ and its right endpoint $\left(\beta,(\sqrt{j}+1)^{2}\right) \in J \times\left\{(\sqrt{j}+1)^{2}\right\}$. Let $\gamma=\left.h_{n}\right|_{[\alpha, \beta]}$.

There is an interval $I^{*}=[\eta, \zeta] \in \mathcal{T}_{j}$ which abuts $J$ on the left; that is, $\zeta$ is the left endpoint of $J$. Clearly, $\eta$ is greater than or equal to the right endpoint of $I$. We know that $\left|h_{n}(x)-1\right|<0.01$ for all $x \in T_{0.01}$ and since 
both $\eta$ and $\zeta$ are in $T_{0.01}$ it follows that the rise of $\gamma$ across $I^{*}$ is less than 0.02 and $\gamma(\zeta)<1.01$. Thus, the rise of $\gamma$ across $[\zeta, \beta]$ is less than $(\beta-\zeta)(.02) / g_{j}<$ $l_{j}(.02) / g_{j}=.025 j$. Consequently, $\gamma(\beta)<1.01+.025 j<j+1<(\sqrt{j}+1)^{2}$, a contradiction. Thus, $F^{2}$ is not UPA.

Before looking at other examples of UPA functions, we wish to point out that we found ourselves having to resort to an unbounded function to exhibit the above behavior. It would be interesting to know if there is a non-negative bounded UPA function whose square is not UPA. More to the point, it would be interesting to know if the class of bounded UPA functions is preserved under outer homeomorphisms and if this class can be characterized in terms of associated sets. We do not know.

Proposition 1. Let $f:[0,1] \rightarrow \mathbb{R}$ be a bounded, Baire 1, interpolatively continuous function with the property that for each natural number $n$ the set

$$
W_{n} \equiv\left\{x: \omega_{f}(x) \geq 1 / n\right\}
$$

is finite. Then $f$ is universally polygonally approximable.

Proof. We shall define a sequence $\left\{h_{n}\right\}$ which $C(f)$-polygonally approximates $f$.

Fix an $n \in \mathbb{N}$. List the elements of $W_{n}$ as $\left\{x_{i}, i=1, \ldots, m_{n}\right\}$, and let $\delta_{n}$ be the smaller of $1 /(n+1)$ and one-half the minimum distance between points in $W_{n}$. Since $f$ is interpolatively continuous at each $x_{i}$, we may select points $y_{i}, z_{i} \in C(f)$ such that $y_{i}<x_{i}<z_{i}, z_{i}-y_{i}<\delta_{1},\left|L_{y_{i}, z_{i}}\left(x_{i}\right)-f\left(x_{i}\right)\right|<1 / n$, and $\omega_{f}\left(\left[y_{i}, z_{i}\right]\right)<2 \omega_{f}\left(x_{i}\right)$. (If $x_{i}=0\left[x_{i}=1\right]$ we modify this selection by taking $y_{i}=x_{i}\left[z_{i}=1\right]$.)

Let $F_{n}=[0,1] \backslash \cup_{i=1}^{m_{n}}\left(y_{i}, z_{i}\right)$. (Here, if $x_{i}=0\left[x_{i}=1\right]$, replace $\left(y_{i}, z_{i}\right)$ by $\left[y_{i}, z_{i}\right)\left[\left(y_{i}, z_{i}\right]\right]$.) For each $x \in F_{n}$ we have $\omega_{f}(x)<1 / n$ and hence we may find an open interval $I_{x}$ containing $x$ for which $\omega_{f}\left(I_{x}\right)<1 / n$. The collection of these $I_{x}$ 's forms an open cover for $F_{n}$ and by extracting a finite subcover, we may select a finite number of points $w_{j} \in\left\{[0,1] \backslash \cup_{i=1}^{m_{n}}\left[y_{i}, z_{i}\right]\right\} \cap C(f)$, $j=1, \ldots, k_{n}$, such that if $h_{n}$ is the polygonal function for $f$ having nodes $0 ; 1 ; w_{j}, j=1, \ldots k_{n} ; y_{i}, i=1, \ldots, m_{n} ; z_{i}, i=1, \ldots, m_{n}$; then for every $x \in W_{n} \cup F_{n}$ we have $\left|h_{n}(x)-f(x)\right|<1 / n$. Note that if $x \in[0,1] \backslash\left(W_{n} \cup F_{n}\right)$, then there is an $x_{i}, i=1, \ldots, m_{n}$ such that $x \in\left[y_{i}, z_{i}\right]$. For such an $x$ we have $h(x)=L_{y_{i}, z_{i}}(x)$, and hence $|h(x)-f(x)|<2 \omega_{f}\left(x_{i}\right)$.

To see that $\left\{h_{n}\right\}$ converges pointwise to $f$, let $\epsilon>0$ and $x \in[0,1]$ be given. If $x \notin C(f)$, then there is an $N>1 / \epsilon$ such that $x \in W_{n}$ for all $n \geq N$ and hence for all $n \geq N,\left|h_{n}(x)-f(x)\right|<1 / n<\epsilon$. On the other hand, suppose $x \in C(f)$. Fix an $N>2 / \epsilon$. There is an $N^{\prime}>N$ such that for all $n>N^{\prime}$ we have $\operatorname{dist}\left(x, W_{N}\right)>\delta_{n}$ and, consequently, $\left|h_{n}(x)-f(x)\right|<2 \cdot \frac{1}{N}<\epsilon$. 
We immediately obtain the following

Corollary 1. Let $f:[0,1] \rightarrow \mathbb{R}$ be an interpolatively continuous function of bounded variation. Then $f$ is universally polygonally approximable.

Corollary 2. Let $f:[0,1] \rightarrow \mathbb{R}$ be a monotone function. Then $f$ is universally polygonally approximable.

Proof. This follows from Corollary 1 and the following lemma.

Lemma 1. If $f:[0,1] \rightarrow \mathbb{R}$ is nondecreasing, then $f$ is interpolatively continuous.

Proof. It will suffice to show that $f$ is interpolatively continuous at each point of discontinuity in $(0,1)$. Let $x_{o}$ be such a point and let $\epsilon>0$. Let $\delta=\min \left\{\epsilon, f\left(x_{o}^{+}\right)-f\left(x_{o}^{-}\right)\right\}$. Let

$$
(\alpha, \beta)=\left(f\left(x_{o}\right)+\delta, f\left(x_{o}\right)-\delta\right) \cap\left(f\left(x_{o}^{-}\right)+\delta / 3, f\left(x_{o}^{+}\right)-\delta / 3\right) .
$$

Choose $y \in\left(x_{o}-\delta, x_{o}\right) \cap C(f)$ so that if $L_{\alpha}=L_{(y, f(y))}^{\left(x_{o}, \alpha\right)}$, then the point $v$ such that $L_{\alpha}(v)=f\left(x_{o}+\delta\right)$ satisfies $v<x_{o}+\delta$. Let $L_{\beta}=L_{(y, f(y))}^{\left(x_{o}, \beta\right)}$. If we can show the existence of a $z \in C(f) \cap\left(x_{o}, x_{o}+\delta\right)$ such that $L_{\alpha}(z)<f(z)<L_{\beta}(z)$, then

$$
L_{\beta}\left(x_{o}\right)<L_{y z}\left(x_{o}\right)<L_{\alpha}\left(x_{o}\right)
$$

and consequently

$$
\left|L_{y z}\left(x_{o}\right)-f\left(x_{o}\right)\right|<\delta \leq \epsilon,
$$

indicating that $f$ is interpolatively continuous at $x_{o}$.

To this end, let $c=\inf \left\{x \in\left(x_{o}, x_{o}+\delta\right): f(x) \leq L_{\alpha}(x)\right\}$. Clearly, $f(c) \leq$ $L_{\alpha}(c)$ and $x_{o}<c<x_{o}+\delta$. Let $a$ and $b$ denote the unique points where $L_{\beta}(a)=f(c)$ and $L_{\alpha}(b)=f(c)$. Then $x_{o}<a<b \leq c<x_{o}+\delta$. Furthermore, for each $x \in(a, b)$ we have

$$
L_{\alpha}(x)<f(x) \leq f(b) \leq f(c)=L_{\beta}(a)<L_{\beta}(x) .
$$

Taking any $z \in C(f) \cap(a, b)$ will complete our proof.

Next we wish to observe that if $f$ is a bounded, Baire 1, and interpolatively continuous function, then its upper semicontinuous envelope, $\bar{f}$, is quasicontinuous and, consequently, universally polygonally approximable. Recall that $\bar{f}$ is defined by

$$
\bar{f}(x)=\lim _{r \downarrow 0} \sup \{f(t):|t-x|<r\},
$$


and is known to be upper semicontinuous for any bounded function $f$. We shall show that for a bounded, Baire 1, interpolatively continuous function $f$, $\bar{f}=f^{*}$, where $f^{*}$ is given by

$$
f^{*}(x)=\limsup _{t \rightarrow x, t \in C(f)} f(t) .
$$

Lemma 2. Let $I$ be an interval and let $f: I \rightarrow \mathbb{R}$ be a bounded, Baire 1, interpolatively continuous function. Then

1. $\bar{f}(x)=f^{*}(x)$ for all $x \in I$.

2. $\bar{f}$ is quasi-continuous on $I$.

Proof. Let $x \in I$. Clearly, $\bar{f}(x) \geq f^{*}(x)$. Suppose $\bar{f}(x)-f^{*}(x)=\epsilon>0$. Let $\left\{q_{n}\right\}$ be a sequence of points in $I$ converging to $x$ such that $\lim _{n \rightarrow \infty} f\left(q_{n}\right)=$ $\bar{f}(x)$. Since $f$ is interpolatively continuous at each $q_{n}$, for each $n$ we may find a point $p_{n} \in C(f) \cap\left(q_{n}-1 / n, q_{n}+1 / n\right)$ such that $f\left(p_{n}\right)>f\left(q_{n}\right)-\epsilon$. Hence, $f^{*}(x) \geq \lim \sup _{n \rightarrow \infty} f\left(p_{n}\right) \geq \lim \sup _{n \rightarrow \infty} f\left(q_{n}\right)-\epsilon \geq \bar{f}(x)-\epsilon$. Thus, 1. holds. Clearly, $f^{*}$ is quasi-continuous and so 2 . follows from 1 .

Thus, we have

Proposition 2. If $f:[0,1] \rightarrow \mathbb{R}$ is bounded, Baire 1, and interpolatively continuous, then its upper semicontinuous envelope, $\bar{f}$, is quasi-continuous and therefore universally polygonally approximable.

We may further utilize Lemma 2 to obtain

Proposition 3. Let $f:[0,1] \rightarrow \mathbb{R}$ be a bounded, Baire 1, interpolatively continuous function with only countably many points of discontinuity. Then $f$ is the uniform limit of a sequence of universally approximable functions.

Proof. As in Proposition 1, for each $n \in \mathbb{N}$ let $W_{n}=\left\{x: \omega_{f}(x) \geq 1 / n\right\}$. Then each $W_{n}$ is a countable closed set and is, consequently, scattered. For each $n$ define $f_{n}:[0,1] \rightarrow \mathbb{R}$ by

$$
f_{n}(x)=\left\{\begin{array}{ll}
f(x), & x \in W_{n} \\
\bar{f}(x), & \text { otherwise }
\end{array} .\right.
$$

Clearly, $\left|f_{n}(x)-f(x)\right| \leq 1 / n$ for all $x \in[0,1]$. On each component interval of the open set $[0,1] \backslash W_{n}$, we have, via Lemma 2 , that $f_{n}$ is upper semicontinuous and quasi-continuous. Since $W_{n}$ is scattered, $f_{n}$ is a Baire 1 function. Also, since $f_{n}=f$ on $C(f), f_{n}$ is interpolatively continuous at each $x \in W_{n}$, and, 
consequently, is interpolatively continuous on all of $(0,1)$. Since $f_{n}$ is Baire 1 , interpolatively continuous, and has $N Q\left(f_{n}\right) \subset W_{n}$, a scattered set, it follows from [5] that $f_{n}$ is universally polygonally approximable.

We will have more to say about the class of uniform limits of universally polygonally approximable functions in Section 4, but hasten to add, once again, that we do not know if previous proposition can be improved to conclude that $f \in \mathrm{UPA}$.

\section{Subclasses of Baire 1}

It was observed in [5] that the class of universally approximable functions is not closed under uniform limits. Here, we wish to explore how the collection of these functions is situated in the class of Baire 1 functions. The basic goal of this section will be to make sense of and verify the correctness of the following diagram.

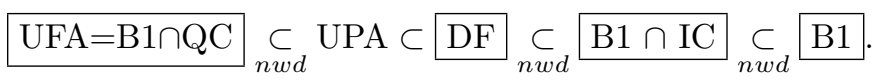

Here, UFA denotes the class of universally first return approachable functions, which were defined in [3]. Also in that paper a proof is given for UFA = $\mathrm{B} 1 \cap \mathrm{QC}$. A box around a class in the diagram (1) indicates that the class is closed under uniform limits and the symbol " $\subset$ " " is to be read "is a nowhere dense subset of" where the sup metric is understood. We will verify the correctness of (1), moving from left to right.

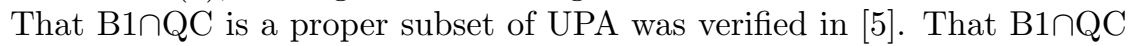
is closed in the sup metric is a straight-forward exercise. We'll observe its validity as a consequence of the following lemma, with which we shall also be able to conclude that, in a porosity sense, "most" points are quasi-continuity points for a function in the closure of UPA.

Lemma 3. Suppose that $\left\{f_{n}\right\}$ is a uniformly convergent sequence of Baire 1 functions on $[0,1]$ with limit $f$ and that each $f_{n}$ is quasi-continuous at $x_{o}$. Then $f$ is quasi-continuous at $x_{o}$.

Proof. Let $\delta$ and $\epsilon$ be given positive numbers. Our goal is to find a $z \in$ $C(f) \cap\left(x_{o}-\delta, x_{o}+\delta\right)$ such that $f(z) \in\left(f\left(x_{o}\right)-\epsilon, f\left(x_{o}\right)+\epsilon\right)$.

First, find $N \in \mathbb{N}$ such that for all $n \geq N$ and all $x \in[0,1]$, we have $\left|f_{n}(x)-f(x)\right|<\epsilon / 3$. Since $f_{N}$ is quasi-continuous at $x_{o}$, there is a second category set $A \subseteq C\left(F_{N}\right) \cap\left(x_{o}-\delta, x_{o}+\delta\right)$ such that for all $x \in A, \mid f_{N}(x)-$ $f_{N}\left(x_{o}\right) \mid<\epsilon / 3$. Since $C(f)$ is residual, there is a $z \in A \cap C(f)$. Then

$$
\left|f(z)-f\left(x_{o}\right)\right| \leq\left|f(z)-f_{N}(z)\right|+\left|f_{N}(z)-f_{N}\left(x_{o}\right)\right|+\left|f_{N}\left(x_{o}\right)-f\left(x_{o}\right)\right|<\epsilon .
$$


Hence, $f$ is quasi-continuous at $x_{o}$.

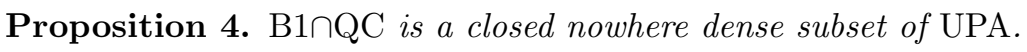

Proof. From the preceding, it only remains to show that B1 $\cap \mathrm{QC}$ is nowhere dense in UPA. For each $g \in$ UPA and $r>0$ let the open ball about $g$ of radius $r$ be denoted by $B(g, r)$; i.e.,

$$
B(g, r)=\{h \in \mathrm{B} 1:\|h-g\|<r\},
$$

where $\|h-g\|=\sup \{|h(x)-g(x)|: x \in[0,1]\}$. Now, let $F \in$ UPA and $\epsilon>0$. Let $\left\{h_{n}\right\}$ be a sequence of polygonal functions for $F$ which $C(F)$-polygonally approximates $F$. Choose $x_{o} \in C(F) \cap(0,1)$ and let $\delta>0$ be such that if $\left|x-x_{o}\right|<\delta$, then $\left|F(x)-F\left(x_{o}\right)\right|<\epsilon / 4$. Choose $u, v$ in $C(F)$ such that $x_{o}-\delta<u<x_{o}<v<x_{o}+\delta$. Note that there is no loss of generality in assuming that $u$ and $v$ are nodes of each $h_{n}$. Define $g:[0,1] \rightarrow \mathbb{R}$ by

$$
g(x)=\left\{\begin{array}{ll}
F(x), & x \in[0, u) \\
L_{(u, F(u))}^{\left(x_{o}, F\left(x_{o}\right)+\epsilon / 4\right)}(x), & x \in\left[u, x_{o}\right) \\
F\left(x_{o}\right), & x=x_{o} \\
L_{\left(x_{o}, F\left(x_{o}\right)-\epsilon / 4\right)}^{(v,(v)),} & x \in\left(x_{o}, v\right] \\
F(x), & x \in(v, 1]
\end{array} .\right.
$$

It is easily seen that $g \in \mathrm{UPA}$ and $g \in B(F, \epsilon / 2)$. Furthermore, $g$ is not quasicontinuous at $x_{o}$. Indeed, if we let $d$ denote one-half the distance from the point $\left(x_{o}, f\left(x_{o}\right)\right)$ to the union of the graphs of $L_{(u, F(u))}^{\left(x_{o}, F\left(x_{o}\right)+\epsilon / 4\right)}$ and $L_{\left(x_{o}, F\left(x_{o}\right)-\epsilon / 4\right)}^{(v, F(v)}$, then no function in $B(g, d)$ can be quasi-continuous at $x_{o}$. We have

$$
B(g, d) \subseteq B(g, \epsilon / 2) \subseteq B(F, \epsilon)
$$

and thus $\mathrm{B} 1 \cap \mathrm{QC}$ is nowhere dense in UPA.

Now, we make a short digression to observe the following:

Proposition 5. If $f:[0,1] \rightarrow \mathbb{R}$ is the uniform limit of a sequence of UPA functions, then $N Q(f)$ is $\sigma-(1-\epsilon)$-symmetrically porous for every $\epsilon>0$.

Proof. In [5] it was shown that if $g \in \mathrm{UPA}$, then $N Q(g)$ is $\sigma-(1-\epsilon)$ symmetrically porous for every $\epsilon>0$. If $f$ is the uniform limit of the sequence $\left\{f_{n}\right\}$ of UPA functions, then Lemma 3 guarantees that $N Q(f) \subseteq \cup_{n=1}^{\infty} N Q\left(f_{n}\right)$ and the proof follows.

Returning to the verification of (1), our next immediate goal is to show that $\mathrm{UPA} \subseteq \mathrm{DF}$. 
Proposition 6. If $f:[0,1] \rightarrow \mathbb{R}$ is universally polygonally approximable then it has the delta-fine property.

Proof. Let $D \subseteq C(f)$ be dense in $[0,1]$ and let $\left\{h_{n}\right\}$ be a sequence of polygonal functions all having vertices on $f \mid D$ which converges pointwise to $f$ on $[0,1]$. Let $W$ be a closed set and $\epsilon>0$. According to the Baire Category Theorem there must be what several authors call a point of uniform convergence of $\left\{h_{n}\right\}$ relative to $W$, e.g., see [6]. In particular, this entails that there is a portion $(c, d) \cap W$ and an $N$ such that for all $n>N$ and all $x \in(c, d) \cap W$ we have $\left|h_{n}(x)-f(x)\right|<\epsilon / 2$. Choose $a<b$ in $(c, d) \cap D$ so that $(a, b) \cap W \neq \emptyset$. Let $\delta>0$ and choose $n>N$ so large that mesh $\left(h_{n}\right)<\delta$ and that both $\left|h_{n}(a)-f(a)\right|$ and $\left|h_{n}(b)-f(b)\right|$ are less than $\epsilon / 2$. Let $p_{n}$ be the polygonal function obtained by using all the vertices of $h_{n}$ plus $(a, f(a))$ and $(b, f(b))$. Then, clearly, $\operatorname{mesh}\left(p_{n}\right)<\delta$ and for all $x \in[a, b] \cap W$ we have $\left|p_{n}(x)-f(x)\right|<\epsilon$, implying that $f$ has the delta-fine property.

In [5] it was shown that UPA is not closed in the sup metric. However, DF is, which can be seen as follows.

Lemma 4. If $f:[0,1] \rightarrow \mathbb{R}$ has the delta-fine property, then for any dense $D \subseteq C(f)$ we have that for each closed set $W$ and each $\epsilon>0$, there are two points $c<d$ in $D$ such that $[c, d] \cap W \neq \emptyset$ and such that for every $\delta>0$ there is a $\delta$-fine partition $\tau=\left\{c=c_{0}<c_{1}<c_{2}<\cdots<c_{n}=d\right\}$ of $[c, d]$ consisting of points in $D$ such that $\left|P_{\tau,[c, d]}(x)-f(x)\right|<\epsilon$ for every $x \in W \cap[c, d]$.

Proof. Let a closed set $W$ and an $\epsilon>0$ be given. Let $a<b$ be two points in $C(f)$ that satisfy the definition of delta-fine using $\epsilon / 3$. Next choose a $\delta_{0}$ such that if $|s-a|<\delta_{0}$ and $|t-b|<\delta_{0}$ then both $|f(a)-f(c)|$ and $|f(b)-f(d)|$ are less than $\epsilon / 3$. Next choose $c, d \in D$ so that $a \leq c<a+\delta_{0}, b-\delta_{0}<d \leq b$, and $[c, d] \cap W \neq \emptyset$.

Now, let $0<\delta \leq \delta_{0}$. Then there is a $\delta / 2$-fine partition $\rho=\left\{a=a_{0}<\right.$ $\left.a_{1}<a_{2}<\cdots<a_{n}=b\right\}$ of $[a, b]$ consisting of points in $C(f)$ such that $\left|P_{\rho,[a, b]}(x)-f(x)\right|<\epsilon / 3$ for every $x \in W \cap[a, b]$. Let $\mu=\{c, d\} \cup(\rho \cap[c, d])$. Then for every $x \in W \cap[c, d]$ we have $\left|P_{\mu,[c, d]}(x)-f(x)\right|<2 \epsilon / 3$. If $\delta^{\prime}$ is the mesh of $\mu$ we replace each element of $\mu \backslash\{c, d\}$ by a point of $D$ within $\delta^{\prime}$ of it and so close that if we denote the resulting partition of $[c, d]$ by $\tau$ we have $\left|P_{\mu,[c, d]}(x)-P_{\tau,[c, d]}(x)\right|<\epsilon / 3$ for all $x \in[c, d]$. Then $\tau$ is $\delta$-fine and $\left|P_{\tau,[c, d]}(x)-f(x)\right|<\epsilon$ for every $x \in W \cap[c, d]$, completing the proof.

Proposition 7. The uniform limit of a sequence of delta-fine functions is delta-fine. 
Proof. Let $\left\{f_{n}\right\}$ be a sequence of functions, each of which has the delta-fine property, which converges uniformly to $f$. Let $D=\cap_{n=1}^{\infty} C\left(f_{n}\right)$. Then $D$ is a dense $G_{\delta}$ subset of $C(f)$. Let $W$ be a closed set and $\epsilon>0$.

First, choose $N$ so large that $\left|f_{N}(x)-f(x)\right|<\epsilon / 3$ for all $x$. According to the above lemma, there are two points $a<b$ in $D$ such that $[a, b] \cap W \neq \emptyset$ and such that for every $\delta>0$ there is a $\delta$-fine partition $\tau=\left\{a=a_{0}<a_{1}<a_{2}<\cdots<\right.$ $\left.a_{n}=b\right\}$ of $[a, b]$ consisting of points in $D$ such that $\left|P_{f_{N}, \tau,[a, b]}(x)-f_{N}(x)\right|<$ $\epsilon / 3$ for every $x \in W \cap[a, b]$. Note that $\left|P_{f_{N}, \tau,[a, b]}(x)-P_{f, \tau,[a, b]}\right|<\epsilon / 3$ for every $x \in W \cap[a, b]$, and, consequently, $\left|P_{f, \tau,[a, b]}(x)-f(x)\right|<\epsilon / 3$ for every $x \in W \cap[a, b]$, completing the proof that $f$ has the delta-fine property.

Next, we show that $\mathrm{DF} \subseteq \mathrm{B} 1 \cap \mathrm{IC}$.

Proposition 8. If $f:[0,1] \rightarrow \mathbb{R}$ has the delta-fine property, then $f$ is Baire 1 and interpolatively continuous.

Proof. To see that $f$ is interpolatively continuous, let $x \in(0,1)$. If $x \in C(f)$, then clearly $x \in I C(f)$. If $x \notin C(f)$, then we apply the delta-fine property with $W=\{x\}$, yielding $x \in I C(f)$.

To see that $f$ is Baire 1 , let $P$ be a perfect set. Let $\epsilon_{k}=1 / k$. First, using the delta-fine property on $P$ with $\epsilon_{1}$, find the corresponding portion $Q=\left[a_{1}, b_{1}\right] \cap P$. Using any $\delta$, find a $\delta$-fine partition $\tau_{1}$ of $\left[a_{1}, b_{1}\right]$ such that $\left|P_{\tau,\left[a_{1}, b_{1}\right]}(x)-f(x)\right|<\epsilon_{1}$ for all $x \in Q$. Pick a partition subinterval that intersects $Q$ in a perfect set and find a point $z \in Q$ belonging to that subinterval. Next, find a $\delta_{1}$ such that the oscillation of $P_{\tau,\left[a_{1}, b_{1}\right]}$ over the interval $\left[z-\delta_{1}, z+\delta_{1}\right]$ is less than $\epsilon_{1}$. Consequently, the oscillation of $f$ over $P_{1}=\left[z-\delta_{1}, z+\delta_{1}\right] \cap Q$ is less than $3 \epsilon_{1}$.

Now, we may repeat this process, starting with the perfect set $P_{1}$ and using $\epsilon_{2}$. Continuing in this manner, there will be a point $s \in \cap_{k=1}^{\infty} P_{k}$ and this point will be a point of continuity of $f \mid P$, yielding that $f$ is Baire 1 .

In order to show DF is nowhere dense in $\mathrm{B} 1 \cap \mathrm{IC}$ we wish to utilize the function $f \in(\mathrm{B} 1 \cap \mathrm{IC}) \backslash \mathrm{UPA}$ which was constructed in Example 2 in [5]. That function had range $\{0,1 / 2,1\}$, assuming the value $1 / 2$ on a certain Cantor set $T$ and being constantly 0 or 1 on contiguous intervals. Very minor editorial modifications of the proof that $f \notin \mathrm{UPA}$ easily yield a proof that $f \notin \mathrm{DF}$. Indeed, it is also easily seen that if $h \in \mathrm{B} 1$ and $\|h-f\|$ is small, say less than $1 / 10$, then $h \notin \mathrm{DF}$. With this observation, we are ready to establish

Proposition 9. DF is nowhere dense in $\mathrm{B} 1 \cap \mathrm{IC}$.

Proof. We utilize the notation and strategy of Proposition 4. Let $F \in \mathrm{B} 1 \cap \mathrm{IC}$ and let $\epsilon>0$. Choose $x_{o} \in C(F) \cap(0,1)$ and let $\delta>0$ be such that if $\left|x-x_{o}\right|<\delta$, then $\left|F(x)-F\left(x_{o}\right)\right|<\epsilon / 2$. Choose $u, v$ in $C(F)$ such that 
$x_{o}-\delta<u<x_{o}<v<x_{o}+\delta$. Then choose $u^{\prime} \in C(F) \cap\left(u, x_{o}\right)$ and $v^{\prime} \in C(F) \cap\left(x_{o}, v\right)$. Letting $f$ be the function noted in the paragraph preceding this proposition, define $h:\left[u^{\prime}, v^{\prime}\right] \rightarrow\left\{F\left(x_{o}\right)-\epsilon / 2, F\left(x_{o}\right), F\left(x_{o}\right)+\epsilon / 2\right\}$ by

$$
h(x)=F\left(x_{o}\right)-\epsilon / 2+f\left(\frac{x-u^{\prime}}{v^{\prime}-u^{\prime}}\right) .
$$

Now, define $g:[0,1] \rightarrow \mathbb{R}$ by

$$
g(x)= \begin{cases}F(x), & x \in[0, u) \\ L_{(u, F(u))}^{\left(u^{\prime}, F\left(x_{o}\right)\right)}(x), & x \in\left[u, u^{\prime}\right) \\ h(x), & x \in\left[u^{\prime}, v^{\prime}\right] . \\ L_{\left(v^{\prime}, F\left(x_{o}\right)\right)}^{(v, F(v))}(x), & x \in\left(v^{\prime}, v\right] \\ F(x), & x \in(v, 1]\end{cases}
$$

Then $g \in(\mathrm{B} 1 \cap \mathrm{IC}) \backslash \mathrm{DF}, B(g, \epsilon / 2) \subseteq B(F, \epsilon)$, and $B(g, \epsilon / 10) \cap D F=\emptyset$, completing the proof.

That $\mathrm{B} 1 \cap \mathrm{IC}$ is closed was established in [5]. Showing that $\mathrm{B} 1 \cap \mathrm{IC}$ is nowhere dense in B1 can be accomplished in a manner similar to that employed in Proposition 9. Instead of patching in a scaled copy of the function $f$ used there, a scaled copy of the characteristic function of a point will work. This completes the verification of diagram (1).

The gnawing uncertainty is whether the closure of UPA is DF and if not, then what is a descriptive characterization of either UPA or its closure?

\section{References}

[1] S. J. Agronsky, J. G. Ceder, T. L. Pearson, Some characterizations of Darboux Baire 1 functions, Real Anal. Exch. 23 (1997-98), 421-429.

[2] A. M. Bruckner, Differentiation of real functions, CRM Monograph Series, Vol. 5, 2nd ed., American Mathematical Society, ISBN 0-8218-6990-6.

[3] U. B. Darji, M. J. Evans and P. D. Humke, First return approachability, J. Math. Anal. Appl. 199 (1996), 545-557.

[4] U. B. Darji, M. J. Evans, and R. J. O'Malley, Universally first return continuous functions, Proc. Amer. Math. Soc. 123 (1995), 2677-2685.

[5] M. J. Evans, P. D. Humke, and R. J. O'Malley, Universally polygonally approximable functions, J. Appl. Analysis (to appear)

[6] C. Goffman, Real functions, Holt, Reinhart, Winston, New York, 1964. 